\title{
Uniqueness results for fully anti-periodic fractional boundary value problems with nonlinearity depending on lower-order derivatives
}

\author{
Ahmed Alsaedi ${ }^{*}$, Bashir Ahmad ${ }^{1}$, Nadia Mohamad ${ }^{1}$ and Sotiris K Ntouyas ${ }^{2}$
}

"Correspondence:

aalsaedi@hotmail.com

1 Department of Mathematics,

Faculty of Science, King Abdulaziz University, P.O. Box 80203, Jeddah,

21589, Saudi Arabia

Full list of author information is

available at the end of the article

\begin{abstract}
We investigate the uniqueness of solutions for fully anti-periodic fractional boundary value problems of order $2<q \leq 3$ with nonlinearity depending on lower-order fractional derivatives. Our results are based on some standard fixed point theorems. The paper concludes with illustrative examples.

MSC: $34 \mathrm{~A} 12 ; 34 \mathrm{~A} 40$

Keywords: differential equations of fractional order; anti-periodic fractional boundary conditions; uniqueness; fixed point
\end{abstract}

\section{Introduction}

In this article, we show the existence of solutions for a fully fractional-order anti-periodic boundary value problem of the form

$$
\begin{aligned}
& { }^{c} D^{q} x(t)=f\left(t, x(t),{ }^{c} D^{r} x(t)\right), \quad t \in[0, T], T>0,2<q \leq 3,0<r \leq 1, \\
& x(0)=-x(T), \quad{ }^{c} D^{p} x(0)=-{ }^{c} D^{p} x(T), \\
& { }^{c} D^{p+1} x(0)=-{ }^{c} D^{p+1} x(T), \quad 0<p<1,
\end{aligned}
$$

where ${ }^{c} D^{q}$ denotes the Caputo fractional derivative of order $q$ and $f$ is a given continuous function.

As a second problem, we will discuss the existence of solutions for the following fractional differential equation with the boundary conditions (1.2):

$$
{ }^{c} D^{q} x(t)=f\left(t, x(t),{ }^{c} D^{r} x(t),{ }^{c} D^{r+1} x(t)\right), \quad t \in[0, T], T>0,2<q \leq 3,0<r \leq 1 .
$$

The present work is motivated by a recent paper [1] in which the problem (1.1)-(1.2) was discussed with the nonlinearity of the type $f(t, x)$. Thus the present paper generalizes the results obtained in [1].

In the last few decades, fractional calculus has evolved as an attractive field of research in view of its extensive applications in basic and technical sciences. Examples can be found

○2014 Alsaedi et al.; licensee Springer. This is an Open Access article distributed under the terms of the Creative Commons Attribution License (http://creativecommons.org/licenses/by/2.0), which permits unrestricted use, distribution, and reproduction in any medium, provided the original work is properly cited. 
in physics, chemistry, biology, economics, control theory, signal and image processing, biophysics, blood flow phenomena, aerodynamics, fitting of experimental data, etc. [2-5].

The subject of boundary value problems of differential equations, having an enriched history, has been progressing at the same pace as before. In the context of fractional boundary value problems, there has been a much development in the last ten years; for instance, see [6-25] and the references cited therein.

In view of the importance of anti-periodic boundary conditions in the mathematical modeling of a variety of physical processes [26-28], the study of anti-periodic boundary value problems has received considerable attention. Some recent work on anti-periodic boundary value problems of fractional order can be found in a series of papers [29-34] and the references therein.

\section{Preliminaries}

We begin this section with some basic concepts $[3,4]$.

Definition 2.1 The Riemann-Liouville fractional integral of order $q$ for a continuous function $g$ is defined as

$$
I^{q} g(t)=\frac{1}{\Gamma(q)} \int_{0}^{t} \frac{g(s)}{(t-s)^{1-q}} d s, \quad q>0
$$

provided the integral exists.

Definition 2.2 For a function $g \in A C^{n-1}([0, \infty), \mathbb{R})$, the Caputo derivative of fractional order $q$ is defined as

$$
{ }^{c} D^{q} g(t)=\frac{1}{\Gamma(n-q)} \int_{0}^{t}(t-s)^{n-q-1} g^{(n)}(s) d s, \quad n-1<q<n, n=[q]+1,
$$

where $[q]$ denotes the integer part of the real number $q$.

Lemma 2.1 [1] For any $y \in C[0, T]$, the unique solution of the linear fractional equation ${ }^{c} D^{q} x(t)=y(t), 0<t<T, 2<q \leq 3$ with anti-periodic boundary conditions (1.2) is given by

$$
x(t)=\int_{0}^{T} G(t, s) y(s) d s
$$

where $G(t, s)$ is the Green's function (depending on $q$ and $p$ ) given by

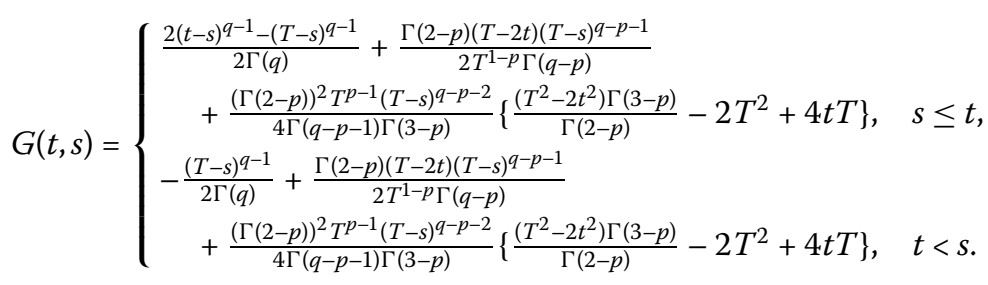

\section{Uniqueness of solutions}

This section is devoted to the uniqueness of solutions for the problems at hand by means of Banach's contraction principle. 


\subsection{Uniqueness result for the problem (1.1)-(1.2)}

For $0<r \leq 1$, let us define a space $\mathcal{C}=\left\{x: x,{ }^{c} D^{r} x \in C([0, T])\right\}$, where $C([0, T])$ denotes the space of all continuous functions defined on $[0, T]$. Note that the space $\mathcal{C}$ endowed with the norm defined by $\|x\|=\sup \left\{|x(t)|+\left|{ }^{c} D^{r} x(t)\right|, t \in[0, T]\right\}$ is a Banach space.

In view of Lemma 2.1, let us define an operator $\mathcal{G}: \mathcal{C} \rightarrow \mathcal{C}$ associated with the problem (1.1)-(1.2) as

$$
\begin{aligned}
(\mathcal{G} x)(t)= & \int_{0}^{t} \frac{(t-s)^{q-1}}{\Gamma(q)} f\left(s, x(s),{ }^{c} D^{r} x(s)\right) d s-\frac{1}{2} \int_{0}^{T} \frac{(T-s)^{q-1}}{\Gamma(q)} f\left(s, x(s),{ }^{c} D^{r} x(s)\right) d s \\
& +\mu(t) \int_{0}^{T} \frac{(T-s)^{q-p-1}}{\Gamma(q-p)} f\left(s, x(s),{ }^{c} D^{r} x(s)\right) d s \\
& +v(t) \int_{0}^{T} \frac{(T-s)^{q-p-2}}{\Gamma(q-p-1)} f\left(s, x(s),{ }^{c} D^{r} x(s)\right) d s,
\end{aligned}
$$

where

$$
\mu(t)=\frac{\Gamma(2-p)(T-2 t)}{2 T^{1-p}}, \quad v(t)=\frac{\Gamma(2-p)\left(T^{2}-2 t^{2}-\frac{2 \Gamma(2-p) T^{2}}{\Gamma(3-p)}+\frac{4 t T \Gamma(2-p)}{\Gamma(3-p)}\right)}{4 T^{1-p}} .
$$

Observe that the problem (1.1)-(1.2) has a solution only if the operator $\mathcal{G}$ has a fixed point.

Before proceeding further, let us introduce some notations:

$$
\mathrm{N}=\max \left\{\mathrm{N}_{1}, \mathrm{~N}_{2}\right\}
$$

where

$$
\begin{aligned}
\mathrm{N}_{1}= & T^{q}\left[\frac{3}{2 \Gamma(q+1)}+\frac{\Gamma(2-p)}{2 \Gamma(q-p+1)}+\frac{\Gamma(2-p)}{4 \Gamma(q-p)}\left[1-\frac{2 \Gamma(2-p)}{\Gamma(3-p)}+2\left(\frac{\Gamma(2-p)}{\Gamma(3-p)}\right)^{2}\right]\right], \\
\mathrm{N}_{2}= & T^{q-r}\left[\frac{1}{\Gamma(q-r+1)}+\frac{\Gamma(2-p)}{\Gamma(2-r) \Gamma(q-p+1)}\right. \\
& \left.+\frac{\Gamma(2-p)}{\Gamma(q-p)}\left[\frac{\Gamma(2-p)}{\Gamma(3-p) \Gamma(2-r)}-\frac{1}{2 \Gamma(3-r)}\right]\right] .
\end{aligned}
$$

Theorem 3.1 Assume that $f:[0, T] \times \mathbb{R} \times \mathbb{R} \rightarrow \mathbb{R}$ is a continuous function satisfying the condition

$$
|f(t, x, \bar{x})-f(t, y, \bar{y})| \leq L(|x-y|+|\bar{x}-\bar{y}|), \quad \forall t \in[0, T], x, y, \bar{x}, \bar{y} \in \mathbb{R}
$$

with $L<\frac{1}{\mathrm{~N}}$, where $\mathrm{N}$ is given by (3.2). Then the anti-periodic boundary value problem (1.1)(1.2) has a unique solution.

Proof Let us set $\sup _{t \in[0, T]}|f(t, 0,0)|=M<\infty$ and $R \geq M \mathrm{~N}(1-L \mathrm{~N})^{-1}$ to show that $\mathcal{G} B_{R} \subset$ $B_{R}$, where $B_{R}=\{x \in \mathcal{C}:\|x\| \leq R\}$. For $x \in B_{R}$, we have

$$
\begin{aligned}
|(\mathcal{G} x)(t)| \leq & \int_{0}^{t} \frac{(t-s)^{q-1}}{\Gamma(q)}\left|f\left(s, x(s),{ }^{c} D^{r} x(s)\right)\right| d s \\
& +\frac{1}{2} \int_{0}^{T} \frac{(T-s)^{q-1}}{\Gamma(q)}\left|f\left(s, x(s),{ }^{c} D^{r} x(s)\right)\right| d s
\end{aligned}
$$




$$
\begin{aligned}
& +|\mu(t)| \int_{0}^{T} \frac{(T-s)^{q-p-1}}{\Gamma(q-p)}\left|f\left(s, x(s),{ }^{c} D^{r} x(s)\right)\right| d s \\
& +|v(t)| \int_{0}^{T} \frac{(T-s)^{q-p-2}}{\Gamma(q-p-1)}\left|f\left(s, x(s),{ }^{c} D^{r} x(s)\right)\right| d s \\
\leq & \int_{0}^{t} \frac{(t-s)^{q-1}}{\Gamma(q)}\left(\left|f\left(s, x(s),{ }^{c} D^{r} x(s)\right)-f(s, 0,0)\right|+|f(s, 0,0)|\right) d s \\
& +\frac{1}{2} \int_{0}^{T} \frac{(T-s)^{q-1}}{\Gamma(q)}\left(\left|f\left(s, x(s),{ }^{c} D^{r} x(s)\right)-f(s, 0,0)\right|+|f(s, 0,0)|\right) d s \\
& +|\mu(t)| \int_{0}^{T} \frac{(T-s)^{q-p-1}}{\Gamma(q-p)}\left(\left|f\left(s, x(s),{ }^{c} D^{r} x(s)\right)-f(s, 0,0)\right|+|f(s, 0,0)|\right) d s \\
& +|v(t)| \int_{0}^{T} \frac{(T-s)^{q-p-2}}{\Gamma(q-p-1)}\left(\left|f\left(s, x(s),{ }^{c} D^{r} x(s)\right)-f(s, 0,0)\right|+|f(s, 0,0)|\right) d s \\
\leq & (L R+M)\left[\int_{0}^{t} \frac{(t-s)^{q-1}}{\Gamma(q)} d s+\frac{1}{2} \int_{0}^{T} \frac{(T-s)^{q-1}}{\Gamma(q)} d s\right. \\
& \left.+|\mu(t)| \int_{0}^{T} \frac{(T-s)^{q-p-1}}{\Gamma(q-p)} d s+|v(t)| \int_{0}^{T} \frac{(T-s)^{q-p-2}}{\Gamma(q-p-1)} d s\right] \\
\leq & (L R+M) \mathrm{N}_{1} \leq(L R+M) \mathrm{N} \leq R .
\end{aligned}
$$

Using the facts ${ }^{c} D^{r} b=0$ ( $b$ is a constant $),{ }^{c} D^{r} t=\frac{t^{1-r}}{\Gamma(2-r)},{ }^{c} D^{r} t^{2}=\frac{2 t^{2-r}}{\Gamma(3-r)},{ }^{c} D^{r+1} t^{2}=\frac{2 t^{1-r}}{\Gamma(2-r)}$, for $0<r<1$, we get

$$
\begin{aligned}
\left({ }^{c} D^{r} \mathcal{G} x\right)(t)= & \int_{0}^{t} \frac{(t-s)^{q-r-1}}{\Gamma(q-r)} f\left(s, x(s),{ }^{c} D^{r} x(s)\right) d s \\
& -\frac{\Gamma(2-p) t^{1-r}}{\Gamma(2-r) T^{1-p}} \int_{0}^{T} \frac{(T-s){ }^{q-p-1}}{\Gamma(q-p)} f\left(s, x(s),{ }^{c} D^{r} x(s)\right) d s \\
& +\Gamma(2-p) T^{p-1}\left[\frac{T t^{1-r} \Gamma(2-p)}{\Gamma(3-p) \Gamma(2-r)}-\frac{t^{2-r}}{\Gamma(3-r)}\right] \\
& \times \int_{0}^{T} \frac{(T-s)^{q-p-2}}{\Gamma(q-p-1)} f\left(s, x(s),{ }^{c} D^{r} x(s)\right) d s .
\end{aligned}
$$

As in the previous step, it can be shown that

$$
\left|\left({ }^{c} D^{r} \mathcal{G} x\right)(t)\right| \leq(L R+M) \mathrm{N}_{2} \leq(L R+M) \mathrm{N} \leq R .
$$

Thus we get $\mathcal{G} x \in B_{R}$. Hence $\mathcal{G} B_{R} \subset B_{R}$. Next, for $x_{1}, x_{2} \in \mathcal{C}$ and for each $t \in[0, T]$, we obtain

$$
\begin{aligned}
& \left|\left(\mathcal{G} x_{1}\right)(t)-\left(\mathcal{G} x_{2}\right)(t)\right| \\
& \leq \int_{0}^{t} \frac{(t-s)^{q-1}}{\Gamma(q)}\left|f\left(s, x_{1},{ }^{c} D^{r} x_{1}\right)-f\left(s, x_{2},{ }^{c} D^{r} x_{2}\right)\right| d s \\
& \quad+\frac{1}{2} \int_{0}^{T} \frac{(T-s)^{q-1}}{\Gamma(q)}\left|f\left(s, x_{1},{ }^{c} D^{r} x_{1}\right)-f\left(s, x_{2},{ }^{c} D^{r} x_{2}\right)\right| d s \\
& \quad+|\mu(t)| \int_{0}^{T} \frac{(T-s)^{q-p-1}}{\Gamma(q-p)}\left|f\left(s, x_{1},{ }^{c} D^{r} x_{1}\right)-f\left(s, x_{2},{ }^{c} D^{r} x_{2}\right)\right| d s
\end{aligned}
$$




$$
\begin{aligned}
& +|v(t)| \int_{0}^{T} \frac{(T-s)^{q-p-2}}{\Gamma(q-p-1)}\left|f\left(s, x_{1},{ }^{c} D^{r} x_{1}\right)-f\left(s, x_{2},{ }^{c} D^{r} x_{2}\right)\right| d s \\
\leq & L\left\|x_{1}-x_{2}\right\|\left[\int_{0}^{t} \frac{(t-s)^{q-1}}{\Gamma(q)} d s+\frac{1}{2} \int_{0}^{T} \frac{(T-s)^{q-1}}{\Gamma(q)} d s\right. \\
& \left.\quad+|\mu(t)| \int_{0}^{T} \frac{(T-s)^{q-p-1}}{\Gamma(q-p)} d s+|v(t)| \int_{0}^{T} \frac{(T-s)^{q-p-2}}{\Gamma(q-p-1)} d s\right] \\
< & L \mathrm{~N}_{1}\left\|x_{1}-x_{2}\right\| \\
\leq & L \mathrm{~N}\left\|x_{1}-x_{2}\right\| .
\end{aligned}
$$

In a similar manner, we find that

$$
\left|\left({ }^{c} D^{r} \mathcal{G} x_{1}\right)(t)-\left({ }^{c} D^{r} \mathcal{G} x_{2}\right)(t)\right| \leq L \mathrm{~N}_{2}\left\|x_{1}-x_{2}\right\| \leq L \mathrm{~N}\left\|x_{1}-x_{2}\right\| .
$$

By the given assumption, $L<1 / \mathrm{N}$, it follows that the operator $\mathcal{G}$ is a contraction. Thus, the conclusion of the theorem follows by the contraction mapping principle (Banach fixed point theorem).

\subsection{Uniqueness result for the problem (1.3)-(1.2)}

Here, we study the uniqueness of solutions for the problem of (1.3)-(1.2). For that, let $\overline{\mathcal{C}}=\left\{x: x,{ }^{c} D^{r} x,{ }^{c} D^{r+1} x(t) \in C([0, T])\right\}$ be a Banach space endowed with the norm $\|x\|=$ $\sup \left\{|x(t)|+\left|{ }^{c} D^{r} x(t)\right|+\left|{ }^{c} D^{r+1} x(t)\right|, t \in[0, T]\right\}, 0<r \leq 1$.

Relative to the problem (1.3)-(1.2), we define an operator $\overline{\mathcal{G}}: \overline{\mathcal{C}} \rightarrow \overline{\mathcal{C}}$ as

$$
\begin{aligned}
(\overline{\mathcal{G}} x)(t)= & \int_{0}^{t} \frac{(t-s)^{q-1}}{\Gamma(q)} f\left(s, x(s),{ }^{c} D^{r} x(s),{ }^{c} D^{r+1} x(s)\right) d s \\
& -\frac{1}{2} \int_{0}^{T} \frac{(T-s)^{q-1}}{\Gamma(q)} f\left(s, x(s),{ }^{c} D^{r} x(s),{ }^{c} D^{r+1} x(s)\right) d s \\
& +\mu(t) \int_{0}^{T} \frac{(T-s)^{q-p-1}}{\Gamma(q-p)} f\left(s, x(s),{ }^{c} D^{r} x(s),{ }^{c} D^{r+1} x(s)\right) d s \\
& +v(t) \int_{0}^{T} \frac{(T-s)^{q-p-2}}{\Gamma(q-p-1)} f\left(s, x(s),{ }^{c} D^{r} x(s),{ }^{c} D^{r+1} x(s)\right) d s .
\end{aligned}
$$

In what follows, we set

$$
\overline{\mathrm{N}}=\max \left\{\mathrm{N}, \mathrm{N}_{3}\right\},
$$

where $\mathrm{N}$ is given by (3.2) and

$$
\mathrm{N}_{3}=T^{q-r-1}\left[\frac{1}{\Gamma(q-r)}+\frac{\Gamma(2-p)}{\Gamma(2-r) \Gamma(q-p)}\right] .
$$

Theorem 3.2 Let $f:[0, T] \times \mathbb{R} \times \mathbb{R} \rightarrow \mathbb{R}$ be a continuous function and there exists a positive number $\bar{L}<1 / \overline{\mathrm{N}}$ such that

$$
|f(t, x, \bar{x}, \overline{\bar{x}})-f(t, y, \bar{y}, \overline{\bar{y}})| \leq \bar{L}(|x-y|+|\bar{x}-\bar{y}|+|\overline{\bar{x}}-\overline{\bar{y}}|), \quad \forall t \in[0, T], x, y, \bar{x}, \bar{y}, \overline{\bar{x}}, \overline{\bar{y}} \in \mathbb{R} .
$$

Then the anti-periodic boundary value problem (1.3)-(1.2) has a unique solution on $[0, T]$. 
Proof We define $B_{\bar{R}}=\{x \in \overline{\mathcal{C}}:\|x\| \leq \bar{R}\}, \bar{R} \geq \frac{\bar{M} \bar{N}}{1-\bar{L} \bar{N}}, \bar{M}=\sup _{t \in[0, T]}|f(t, 0,0,0)|<\infty$ and show that $\overline{\mathcal{G}} B_{\bar{R}} \subset B_{\bar{R}}$. In view of the given assumption, we have

$$
\begin{aligned}
& \left|f\left(s, x(s),{ }^{c} D^{r} x(s),{ }^{c} D^{r+1} x(t)\right)\right| \\
& \quad \leq\left|f\left(s, x(s),{ }^{c} D^{r} x(s),{ }^{c} D^{r+1} x(t)\right)-f(t, 0,0,0)\right|+|f(t, 0,0,0)| \\
& \quad \leq \bar{L} \bar{R}+\bar{M}, \quad x \in B_{\bar{R}} .
\end{aligned}
$$

Thus

$$
\begin{aligned}
|(\overline{\mathcal{G}} x)(t)| \leq & \int_{0}^{t} \frac{(t-s)^{q-1}}{\Gamma(q)}\left|f\left(s, x(s),{ }^{c} D^{r} x(s),{ }^{c} D^{r+1} x(t)\right)\right| d s \\
& +\frac{1}{2} \int_{0}^{T} \frac{(T-s)^{q-1}}{\Gamma(q)}\left|f\left(s, x(s),{ }^{c} D^{r} x(s),{ }^{c} D^{r+1} x(t)\right)\right| d s \\
& +|\mu(t)| \int_{0}^{T} \frac{(T-s)^{q-p-1}}{\Gamma(q-p)}\left|f\left(s, x(s),{ }^{c} D^{r} x(s),{ }^{c} D^{r+1} x(t)\right)\right| d s \\
& +|v(t)| \int_{0}^{T} \frac{(T-s)^{q-p-2}}{\Gamma(q-p-1)}\left|f\left(s, x(s),{ }^{c} D^{r} x(s),{ }^{c} D^{r+1} x(t)\right)\right| d s \\
\leq & (\bar{L} \bar{R}+\bar{M})\left[\int_{0}^{t} \frac{(t-s)^{q-1}}{\Gamma(q)} d s+\frac{1}{2} \int_{0}^{T} \frac{(T-s)^{q-1}}{\Gamma(q)} d s\right. \\
& \left.+|\mu(t)| \int_{0}^{T} \frac{(T-s)^{q-p-1}}{\Gamma(q-p)} d s+|v(t)| \int_{0}^{T} \frac{(T-s)^{q-p-2}}{\Gamma(q-p-1)} d s\right] \\
\leq & (\bar{L} \bar{R}+\bar{M}) \mathrm{N} \\
\leq & (\bar{L} \bar{R}+\bar{M}) \overline{\mathrm{N}} \leq \bar{R} .
\end{aligned}
$$

Further, it can be shown in a similar way that

$$
\begin{aligned}
& \left|\left({ }^{c} D^{r} \overline{\mathcal{G}} x\right)(t)\right| \leq(\bar{L} \bar{R}+\bar{M}) \mathrm{N}_{2} \leq(\bar{L} \bar{R}+\bar{M}) \overline{\mathrm{N}} \leq \bar{R}, \\
& \left|\left({ }^{c} D^{r+1} \overline{\mathcal{G}} x\right)(t)\right| \leq(\bar{L} \bar{R}+\bar{M}) \mathrm{N}_{3} \leq(\bar{L} \bar{R}+\bar{M}) \overline{\mathrm{N}} \leq \bar{R} .
\end{aligned}
$$

Next, for $x_{1}, x_{2} \in \overline{\mathcal{C}}$ and for each $t \in[0, T]$, we obtain

$$
\begin{aligned}
& \left|\left(\overline{\mathcal{G}} x_{1}\right)(t)-\left(\overline{\mathcal{G}} x_{2}\right)(t)\right| \\
& \leq \int_{0}^{t} \frac{(t-s)^{q-1}}{\Gamma(q)}\left|f\left(s, x_{1},{ }^{c} D^{r} x_{1},{ }^{c} D^{r+1} x_{1}\right)-f\left(s, x_{2},{ }^{c} D^{r} x_{2},{ }^{c} D^{r+1} x_{2}\right)\right| d s \\
& \quad+\frac{1}{2} \int_{0}^{T} \frac{(T-s)^{q-1}}{\Gamma(q)}\left|f\left(s, x_{1},{ }^{c} D^{r} x_{1},{ }^{c} D^{r+1} x_{1}\right)-f\left(s, x_{2},{ }^{c} D^{r} x_{2},{ }^{c} D^{r+1} x_{2}\right)\right| d s \\
& \quad+|\mu(t)| \int_{0}^{T} \frac{(T-s)^{q-p-1}}{\Gamma(q-p)}\left|f\left(s, x_{1},{ }^{c} D^{r} x_{1},{ }^{c} D^{r+1} x_{1}\right)-f\left(s, x_{2},{ }^{c} D^{r} x_{2},{ }^{c} D^{r+1} x_{2}\right)\right| d s \\
& \quad+|v(t)| \int_{0}^{T} \frac{(T-s)^{q-p-2}}{\Gamma(q-p-1)}\left|f\left(s, x_{1},{ }^{c} D^{r} x_{1},{ }^{c} D^{r+1} x_{1}\right)-f\left(s, x_{2},{ }^{c} D^{r} x_{2},{ }^{c} D^{r+1} x_{2}\right)\right| d s \\
& \leq L\left\|x_{1}-x_{2}\right\|\left[\int_{0}^{t} \frac{(t-s)^{q-1}}{\Gamma(q)} d s+\frac{1}{2} \int_{0}^{T} \frac{(T-s)^{q-1}}{\Gamma(q)} d s\right.
\end{aligned}
$$




$$
\begin{aligned}
& \left.+|\mu(t)| \int_{0}^{T} \frac{(T-s)^{q-p-1}}{\Gamma(q-p)} d s+|v(t)| \int_{0}^{T} \frac{(T-s)^{q-p-2}}{\Gamma(q-p-1)} d s\right] \\
< & \bar{L} \mathrm{~N}_{1}\left\|x_{1}-x_{2}\right\| \\
\leq & \bar{L} \overline{\mathrm{N}}\left\|x_{1}-x_{2}\right\| .
\end{aligned}
$$

Also, we have

$$
\begin{aligned}
& \left|\left({ }^{c} D^{r} \overline{\mathcal{G}} x_{1}\right)(t)-\left({ }^{c} D^{r} \overline{\mathcal{G}} x_{2}\right)(t)\right| \leq L \mathrm{~N}_{2}\left\|x_{1}-x_{2}\right\| \leq \bar{L} \overline{\mathrm{N}}\left\|x_{1}-x_{2}\right\|, \\
& \left|\left({ }^{c} D^{r+1} \overline{\mathcal{G}} x_{1}\right)(t)-\left({ }^{c} D^{r+1} \overline{\mathcal{G}} x_{2}\right)(t)\right| \leq \bar{L} \mathrm{~N}_{3}\left\|x_{1}-x_{2}\right\| \leq \bar{L} \overline{\mathrm{N}}\left\|x_{1}-x_{2}\right\| .
\end{aligned}
$$

Since $\bar{L}<1 / \overline{\mathrm{N}}$, therefore, the operator $\overline{\mathcal{G}}$ is a contraction. Thus, it follows by the contraction mapping principle that the problem (1.3)-(1.2) has a unique solution on $[0, T]$.

\section{Examples}

(a) Consider the anti-periodic fractional boundary value problem given by

$$
\begin{aligned}
& { }^{c} D^{\frac{5}{2}} x(t)=L\left(\frac{|x(t)|}{1+|x(t)|}+\frac{1}{\sqrt{1+t}}\left(\frac{\left|{ }^{c} D^{\frac{1}{2}} x(t)\right|}{1+\left|{ }^{c} D^{\frac{1}{2}} x(t)\right|}\right)\right)+\sqrt{1+\sin ^{3} t}, \quad L>0, t \in[0,1], \\
& x(0)=-x(1), \quad{ }^{c} D^{\frac{1}{2}} x(0)=-{ }^{c} D^{\frac{1}{2}} x(1), \quad{ }^{c} D^{\frac{3}{2}} x(0)=-{ }^{c} D^{\frac{3}{2}} x(1),
\end{aligned}
$$

where $q=5 / 2, p=1 / 2, r=1 / 2, T=1$, and

$$
f(t, x, \bar{x})=L\left(\frac{|x|}{1+|x|}+\frac{1}{\sqrt{1+t}}\left(\frac{|\bar{x}|}{1+|\bar{x}|}\right)\right)+\sqrt{1+\sin ^{3} t}, \quad \bar{x}={ }^{c} D^{\frac{1}{2}} x(t) .
$$

Clearly

$$
\begin{aligned}
& |f(t, x, \bar{x})-f(t, y, \bar{y})| \leq L\left(\left|\frac{x}{1+x}-\frac{y}{1+y}\right|+\left|\frac{\bar{x}}{1+\bar{x}}-\frac{\bar{y}}{1+\bar{y}}\right|\right) \leq L(|x-y|+|\bar{x}-\bar{y}|), \\
& \mathrm{N}_{1}=\frac{4}{5 \sqrt{\pi}}+\frac{7 \sqrt{\pi}}{36}, \quad \mathrm{~N}_{2}=\frac{5}{3} .
\end{aligned}
$$

With $L<1 / \mathrm{N}\left(\mathrm{N}=\mathrm{N}_{2}>\mathrm{N}_{1}\right)$, all the assumptions of Theorem 3.1 hold. Therefore, the problem (4.1) has a unique solution on $[0,1]$.

(b) Consider the following anti-periodic fractional boundary value problem:

$$
\begin{aligned}
&{ }^{c} D^{\frac{5}{2}} x(t)= \bar{L}\left(\frac{|x(t)|}{1+|x(t)|}+\tan ^{-1}\left|{ }^{c} D^{\frac{1}{2}} x(t)\right|+\cos t\left(\frac{\left|{ }^{c} D^{\frac{3}{2}} x(t)\right|}{1+\left|{ }^{c} D^{\frac{3}{2}} x(t)\right|}\right)\right) \\
&+e^{-t}, \quad \bar{L}>0, t \in[0,1], \\
& x(0)=-x(1), \quad{ }^{c} D^{\frac{1}{2}} x(0)=-{ }^{c} D^{\frac{1}{2}} x(1), \quad{ }^{c} D^{\frac{3}{2}} x(0)=-{ }^{c} D^{\frac{3}{2}} x(1),
\end{aligned}
$$

where $q=5 / 2, p=3 / 4, r=1 / 2, T=1$. With $\bar{x}={ }^{c} D^{\frac{1}{2}} x(t), \overline{\bar{x}}={ }^{c} D^{\frac{3}{2}} x(t)$, we can write

$$
f(t, x, \bar{x}, \overline{\bar{x}})=\bar{L}\left(\frac{|x|}{1+|x|}+\tan ^{-1}|(\bar{x})|+\cos t\left(\frac{|\overline{\bar{x}}|}{1+|\overline{\bar{x}}|}\right)\right)+e^{-t}
$$


Furthermore, we have

$$
\begin{aligned}
& |f(t, x, \bar{x}, \overline{\bar{x}})-f(t, y, \bar{y}, \overline{\bar{y}})| \leq \bar{L}(|x-y|+|\bar{x}-\bar{y}|+\mid \overline{\bar{x}}-\overline{\bar{y}}), \\
& \mathrm{N}_{1}=\frac{4}{5 \sqrt{\pi}}-\frac{73 \Gamma(1 / 4)}{525 \Gamma(3 / 4)}, \quad \mathrm{N}_{2}=\frac{1}{2}+\frac{61 \Gamma(1 / 4)}{105 \sqrt{\pi} \Gamma(3 / 4)}, \quad \mathrm{N}_{3}=1+\frac{2 \Gamma(1 / 4)}{3 \sqrt{\pi} \Gamma(3 / 4)} .
\end{aligned}
$$

Clearly all the assumptions of Theorem 3.2 are satisfied with $\bar{L}<1 / \overline{\mathrm{N}}\left(\overline{\mathrm{N}}=\mathrm{N}_{3}>\mathrm{N}_{2}>\mathrm{N}_{1}\right)$. Hence, the problem (4.2) has a unique solution on $[0,1]$.

\section{Competing interests}

The authors declare that they have no competing interests.

\section{Authors' contributions}

Each of the authors, AA, BA, NM, and SKN, contributed to each part of this work equally and read and approved the final version of the manuscript.

\section{Author details}

'Department of Mathematics, Faculty of Science, King Abdulaziz University, P.O. Box 80203, Jeddah, 21589, Saudi Arabia.

${ }^{2}$ Department of Mathematics, University of Ioannina, loannina, 451 10, Greece.

\section{Authors' information}

Sotiris K Ntouyas is a member of Nonlinear Analysis and Applied Mathematics (NAAM) - Research Group at King Abdulaziz University, Jeddah, Saudi Arabia.

\section{Acknowledgements}

This research was partially supported by the Deanship of Scientific Research (DSR), King Abdulaziz University, Jeddah Saudi Arabia.

Received: 20 February 2014 Accepted: 2 April 2014 Published: 07 May 2014

\section{References}

1. Ahmad, B, Nieto, JJ, Alsaedi, A, Mohamad, N: On a new class of anti-periodic fractional boundary value problems. Abstr. Appl. Anal. 2013, Article ID 606454 (2013)

2. Baleanu, D, Diethelm, K, Scalas, E, Trujillo, JJ: Fractional Calculus Models and Numerical Methods. Series on Complexity, Nonlinearity and Chaos. World Scientific, Boston (2012)

3. Kilbas, AA, Srivastava, HM, Trujillo, JJ: Theory and Applications of Fractional Differential Equations. North-Holland Mathematics Studies, vol. 204. Elsevier, Amsterdam (2006)

4. Podlubny, I: Fractional Differential Equations. Academic Press, San Diego (1999)

5. Sabatier, J, Agrawal, OP, Machado, JAT (eds.): Advances in Fractional Calculus: Theoretical Developments and Applications in Physics and Engineering. Springer, Dordrecht (2007)

6. Agarwal, RP, Andrade, B, Cuevas, C: Weighted pseudo-almost periodic solutions of a class of semilinear fractional differential equations. Nonlinear Anal., Real World Appl. 11, 3532-3554 (2010)

7. Agarwal, RP, Ntouyas, SK, Ahmad, B, Alhothuali, MS: Existence of solutions for integro-differential equations of fractional order with nonlocal three-point fractional boundary conditions. Adv. Differ. Equ. 2013, 128 (2013)

8. Ahmad, B: On nonlocal boundary value problems for nonlinear integro-differential equations of arbitrary fractional order. Results Math. 63, 183-194 (2013)

9. Bai, ZB: On positive solutions of a nonlocal fractional boundary value problem. Nonlinear Anal. 72, 916-924 (2010)

10. Bai, C: Existence of solutions for a nonlinear fractional boundary value problem via a local minimum theorem. Electron. J. Differ. Equ. 2012, 176 (2012)

11. Caballero, J, Harjani, J, Sadarangani, K: On existence and uniqueness of positive solutions to a class of fractional boundary value problems. Bound. Value Probl. 2011, 25 (2011)

12. Chen, F: Coincidence degree and fractional boundary value problems with impulses. Comput. Math. Appl. 64 3444-3455 (2012)

13. Ding, $X-L$, Jiang, $Y$-L: Analytical solutions for the multi-term time-space fractional advection-diffusion equations with mixed boundary conditions. Nonlinear Anal., Real World Appl. 14, 1026-1033 (2013)

14. Ford, NJ, Morgado, ML: Fractional boundary value problems: analysis and numerical methods. Fract. Calc. Appl. Anal. 14(4), 554-567 (2011)

15. Gafiychuk, V, Datsko, B, Meleshko, V: Mathematical modeling of different types of instabilities in time fractional reaction-diffusion systems. Comput. Math. Appl. 59, 1101-1107 (2010)

16. Graef, JR, Kong, L, Kong, Q, Wang, M: Uniqueness of positive solutions of fractional boundary value problems with non-homogeneous integral boundary conditions. Fract. Calc. Appl. Anal. 15, 509-528 (2012)

17. Graef, JR, Kong, L, Yang, B: Positive solutions for a semipositone fractional boundary value problem with a forcing term. Fract. Calc. Appl. Anal. 15, 8-24 (2012)

18. Wang, G, Liu, S, Baleanu, D, Zhang, L: Existence results for nonlinear fractional differential equations involving different Riemann-Liouville fractional derivatives. Adv. Differ. Equ. 2013, 280 (2013)

19. Hernández, E, O'Regan, D, Balachandran, K: Existence results for abstract fractional differential equations with nonlocal conditions via resolvent operators. Indag. Math. 24, 68-82 (2013) 
20. Jarad, F, Abdeljawad, T, Baleanu, D: Stability of $q$-fractional non-autonomous systems. Nonlinear Anal., Real World Appl. 14, 780-784 (2013)

21. Lizama, C: Solutions of two-term time fractional order differential equations with nonlocal initial conditions. Electron. J. Qual. Theory Differ. Equ. 2012, 82 (2012)

22. Machado, JT, Galhano, AM, Trujillo, JJ: Science metrics on fractional calculus development since 1966. Fract. Calc. Appl. Anal. 16, 479-500 (2013)

23. Sun, H-R, Zhang, Q-G: Existence of solutions for a fractional boundary value problem via the mountain pass method and an iterative technique. Comput. Math. Appl. 64, 3436-3443 (2012)

24. Wang, Y, Liu, L, Wu, Y: Positive solutions for a class of fractional boundary value problem with changing sign nonlinearity. Nonlinear Anal. 74, 6434-6441 (2011)

25. Zhao, X, Chai, C, Ge, W: Existence and nonexistence results for a class of fractional boundary value problems. J. Appl. Math. Comput. 41, 17-31 (2013)

26. Aubertin, M, Henneron, T, Piriou, F, Guerin, P, Mipo, J-C: Periodic and anti-periodic boundary conditions with the Lagrange multipliers in the FEM. IEEE Trans. Magn. 46, 3417-3420 (2010)

27. Cardy, JL: Finite-size scaling in strips: antiperiodic boundary conditions. J. Phys. A, Math. Gen. 17, L961-L964 (1984)

28. Chen, Y, Nieto, JJ, O’Regan, D: Anti-periodic solutions for evolution equations associated with maximal monotone mappings. Appl. Math. Lett. 24, 302-307 (2011)

29. Ahmad, B, Nieto, JJ: Existence of solutions for anti-periodic boundary value problems involving fractional differential equations via Leray-Schauder degree theory. Topol. Methods Nonlinear Anal. 35, 295-304 (2010)

30. Ahmad, B, Nieto, JJ: Anti-periodic fractional boundary value problem with nonlinear term depending on lower order derivative. Fract. Calc. Appl. Anal. 15, 451-462 (2012)

31. Benchohra, M, Hamidi, N, Henderson, J: Fractional differential equations with anti-periodic boundary conditions. Numer. Funct. Anal. Optim. 34, 404-414 (2013)

32. Fang, W, Zhenhai, L: Anti-periodic fractional boundary value problems for nonlinear differential equations of fractional order. Adv. Differ. Equ. 2012, 116 (2012)

33. Wang, G, Ahmad, B, Zhang, L: Impulsive anti-periodic boundary value problem for nonlinear differential equations of fractional order. Nonlinear Anal. 74, 792-804 (2011)

34. Wang, X, Guo, X, Tang, G: Anti-periodic fractional boundary value problems for nonlinear differential equations of fractional order. J. Appl. Math. Comput. 41, 367-375 (2013)

10.1186/1687-1847-2014-136

Cite this article as: Alsaedi et al.: Uniqueness results for fully anti-periodic fractional boundary value problems with nonlinearity depending on lower-order derivatives. Advances in Difference Equations 2014, 2014:136

\section{Submit your manuscript to a SpringerOpen ${ }^{\circ}$ journal and benefit from:}

- Convenient online submission

- Rigorous peer review

- Immediate publication on acceptance

- Open access: articles freely available online

- High visibility within the field

- Retaining the copyright to your article 